\title{
The Cross Operator and the Singular Value Analysis for Nonlinear Symmetric Systems
}

\author{
Tudor C. Ionescu \\ Industrial Technology and Management \\ Fac. Math. \& Natural Sciences \\ University of Groningen \\ Nijenborgh 4, 9747 AG Groningen \\ t.c.ionesculrug.nl
}

\author{
Kenji Fujimoto \\ Dept. of Mechanical Science and Engineering \\ Nagoya University \\ Furo-cho, Chikusa-ku, Nagoya 464-8603, Japan \\ E-mail: fujimoto@nagoya-u.jp
}

\author{
Jacquelien M. A. Scherpen \\ Industrial Technology and Management \\ Fac. Math. \& Natural Sciences \\ University of Groningen \\ Nijenborgh 4, 9747 AG Groningen \\ j.m.a.scherpen@rug.nl
}

\begin{abstract}
In this paper, we introduce the notions of statespace symmetry for nonlinear systems and of the cross operator as a nontrivial natural extension of the linear symmetric case, in terms of the controllability and observability operators associated to it. We give a characterization of a symmetric nonlinear system in terms of the cross operator and a coordinate transformation. Then we analyze the use of the cross operator for solving the Hankel singular value problem of the system. The result is a new and simpler characterization of the solutions of this problem in terms of the cross operator and a metric.
\end{abstract}

Index Terms - symmetry, nonlinear systems, cross operator, Hankel, singular value, balancing.

\section{INTRODUCTION}

From a control systems point of view, balanced model order reduction is one of the most popular, attractive and used techniques. It was first introduced by Moore [1] for the linear time-invariant systems case and naturally extended in e.g. [2], [3] for the case of nonlinear systems. In a nutshell, the balanced truncation procedure for a stable minimal nonlinear system, consists of several steps: the first is the computation of the controllability and observability energy functions (Gramians in the linear case), the second is the construction of a coordinate transformation that brings the energy functions into an input normal output diagonal form. The diagonal elements are called the (coordinate free) axis singular value functions of the system and they are invariants, e.g. [3], [5]. The axis singular value functions correspond to the nonlinear version of the singular values of the Hankel operator associated to the system. These values represent the energy measure of the states, telling how "non-minimal" a state is. The truncation consists of ridding the less minimal states of the system. The result is a lower order model that is stable again. In the linear case, there exists an upper bound for the error of approximation, e.g. [6]. One of the drawbacks of balancing for nonlinear systems technique lies in the first step, i.e. the computation of the energy functions which are solutions of Hamilton-Jacobi equations for the nonlinear case and Lyapunov equations in the linear case. In [7], [8], [9], [4] the symmetry of systems is taken into account, to overcome the above mentioned obstacle. This property is encountered especially in (reciprocal) electrical networks as well as in electromechanical systems as well as certain dissipative systems. Symmetry has several definitions that in the linear case can be proven to be equivalent. For instance a class of symmetric systems are the gradient systems, expressed as the gradient with respect to a metric of certain potential functions, see [10] for more details. However, as described in [11], a nonlinear balancing technique adapted for this class of systems is far from being achieved. From a model reduction, state-space point of view a notion of symmetric systems was introduced by Fernando and Nicholson in [12], [13], [14]: a linear system is symmetric if its transfer matrix is symmetric. A minimal LTI system is then symmetric if there exists a linear metric such that the system and its dual are equivalent. In this case there is no need to compute both the controllability and observability Gramians, but only a socalled cross-Gramian that contains information about both the controllability and the observability of the system. It is the solution of a Sylvester equation. The remarkable property is that the absolute value of the eigenvalues of this crossGramian are the Hankel singular values of the system. Used for model reduction, as in [15] a (almost) balanced reduced order model can be obtained. The purpose of this paper is to find a natural extension of the state-space symmetry property as described in [12] and a (non-trivial) extension of the cross-Gramian to a cross-operator, and a similar property as in the linear case, e.g. the (axis) singular value functions to be obtained using the cross operator and simplifying the balancing procedure.

The paper is organized as follows: in Section 2, we give an overview of the linear symmetric systems case, definition the cross-Gramian and properties and present the relation of the cross-Gramian with the controllability and observability operators. In Section 3, we treat the nonlinear case, where we first give an overview of the nonlinear balancing technique related notions and a property of the controllability operator and its derivative. Then we present the definition of a nonlinear symmetric system and of the cross-operator. We also present here a nonlinear version of the Sylvester equation as well as a necessary and sufficient condition for symmetry. In Section 4, we use the cross-operator to solve the nonlinear Hankel differential eigenstructure problem that yields the singular value functions. We conclude the paper 
with open problems and future work ideas.

\section{LINEAR SYMMETRIC SYSTEMS AND THE CROSS GRAMIAN}

In this section we give a brief overview of the model reduction based on the cross-Gramian for a linear symmetric system. First we give the definitions of a linear symmetric system.

Definition 1: [12], [16], [17] Let $G(s)$ be the boundedinput-bounded-output stable transfer matrix of a linear system. It is called symmetric if $G(s)=G^{T}(s)$.

Let

$$
\left\{\begin{array}{l}
\dot{x}=A x+B u \\
y=C x
\end{array}\right.
$$

be a square, minimal asymptotically stable realization of $G(s)$. Then we can give the following characterization:

Proposition 2: [12], [16] (1) is symmetric if and only if there exists an invertible symmetric matrix $T$ such that: $T A=A^{T} T, T B=C^{T}$.

We turn our attention to the definition of the cross Gramian for a linear square system. We have:

Definition 3: [16] Let (1) be a square linear system. Then the cross Gramian $X$ is defined as the solution of the Sylvester equation:

$$
A X+X A+B C=0 .
$$

If the system is asymptotically stable, then the cross Gramian can be defined as: $X=\int_{0}^{\infty} e^{A t} B C e^{A t} d t$.

For the non-symmetric case, the cross Gramian possesses some interesting properties being related to the Hankel operator and the Hankel singular values of a linear square system.

Theorem 4: [16] For square linear systems the non-zero eigenvalues of the cross Gramian $X$ are the non-zero eigenvalues of the Hankel operator associated to the system.

In the symmetric case, see for instance [12], [14], [16], the cross Gramian exhibits some remarkable properties, which are summarized in the sequel.

Defining the controllability Gramian as $W$ and the observability Gramian as $M$, they are the solutions of the following Lyapunov equations, respectively:

$$
\begin{aligned}
& A W+W A^{T}+B B^{T}=0 \\
& A^{T} M+M A+C^{T} C=0 .
\end{aligned}
$$

Theorem 5: [16], [12] Let (1) be a square asymptotically stable symmetric system in the sense of Definition 1 . If $X$ is the solution of (2) then the following relations are equivalent:

1) $X^{2}=W M>0$;

2) If $T=T^{T}$ is the symmetry transformation as in Proposition 2, then: $X=W T=T^{-1} M$;

3 ) the Hankel singular values of (1) are the absolute values of the eigenvalues of $X$.

The properties described in Theorem 5 can be easily rewritten in terms of the controllability, observability and
Hankel operators. We focus our attention on the aforementioned approach since it paves the way for the definitions for nonlinear systems to be presented in Section 3. For the asymptotically stable, minimal system (1) we define the controllability and observability operators, respectively, as:

$$
x_{0}=\mathcal{C}(u)=\int_{0}^{\infty} e^{A t} B u(t) d t, \quad y=\mathcal{O}\left(x_{0}\right)=C e^{A t} x_{0} .
$$

These linear operators admit adjoint counterparts defined as:

$$
\begin{aligned}
& y_{p}=\mathcal{C}^{*}\left(p_{0}\right)=B^{T} e^{A^{T} t} p_{0}, \\
& p_{0}=\mathcal{O}^{*}\left(u_{p}\right)=\int_{0}^{\infty} e^{A^{T} t} B^{T} u_{p} d t .
\end{aligned}
$$

The symmetry property described in Proposition 2 becomes:

Proposition 6: A linear minimal and stable system is symmetric if and only if there exists an invertible and symmetric matrix $T$ such that:

$$
\mathcal{O}^{*}(u)=T \mathcal{C}(u), \mathcal{C}^{*}\left(T x_{0}\right)=\mathcal{O}\left(x_{0}\right) .
$$

If $X$ is the cross Gramian as in Definition 3 and $\mathcal{C}$ and $\mathcal{O}$ are the controllability and observability operators of the system, we have

$$
X x_{0}=\mathcal{C O}\left(x_{0}\right), \text { and } X^{2} x_{0}=\mathcal{C O C O}\left(x_{0}\right)
$$

for all $x_{0}$.

We now rewrite the properties given in Theorem 5 in terms of $\mathcal{C}, \mathcal{O}$ and their adjoints. Although it might seem trivial, this line of thinking is important for the nonlinear extension of the cross Gramian and its properties in Section III-B.2.

Corollary 7: Assume system (1) is asymptotically stable, minimal and symmetric. Then, the eigenvalues of the operator $\mathcal{C O C O}$ are the squared Hankel singular values of the system.

Remark 8: The result of Proposition 7 can be rewritten in many forms, due to the symmetry property, e.g. the Hankel singular values of the system are the eigenvalues of $\mathcal{C C}^{*} T \mathcal{C O}$.

In the next section we will extend the results of Proposition 6 to the nonlinear case in order to define the state-space symmetry property and the cross-operator for a nonlinear dynamical system. The extension of Propositions 6 and 7 will give a relation between the cross-operator and the Hankel eigenstructure problem, namely, the solution of the problem will be given in terms of the properties of the crossoperator.

\section{NONLINEAR CASE}

\section{A. Preliminaries}

We consider the following nonlinear square system:

$$
\begin{aligned}
& \dot{x}=f(x)+g(x) u \\
& y=h(x)
\end{aligned}
$$

with $x \in \mathbb{R}^{n}, u, y \in \mathbb{R}^{m}$, that satisfies the following assumptions: 
1) $f(x), g(x), h(x)$ are smooth functions;

2) 0 is an asymptotically stable equilibrium point and $h(0)=0$

3) system (7) is asymptotically reachable from 0 ;

4) system (7) is zero-state observable.

We define energy functions, certain operators and their state-space representations according to [2], [18], [3], [5]. The following controllability and observability operators are defined for (7), respectively:

$$
\begin{aligned}
& \mathcal{C}: L_{2}^{m}\left(\mathbb{R}_{+}\right) \rightarrow \mathbb{R}^{n}, \mathcal{C}(u)=x_{0}, \\
& \left\{\begin{array}{l}
\dot{x}=-f(x)-g(x) u \quad x(\infty)=0 \\
x_{0}=x(0)
\end{array}\right. \\
& \mathcal{O}: \mathbb{R}^{n} \rightarrow L_{2}^{m}\left(\mathbb{R}_{+}\right), \mathcal{O}\left(x_{0}\right)=y, \\
& \left\{\begin{array}{l}
\dot{x}=f(x) \quad x(\infty)=0 \quad x(0)=x_{0} \\
y=h(x)
\end{array}\right.
\end{aligned}
$$

and the Hankel operator $\mathcal{H}: L_{2}^{m}\left(\mathbb{R}_{+}\right) \rightarrow L_{2}^{m}\left(\mathbb{R}_{+}\right)$for (7) is described by:

$$
\mathcal{H}(u)=\mathcal{O C}(u)
$$

The controllability and observability energy functions are given by:

$$
\begin{aligned}
& L_{c}\left(x_{0}\right)=\frac{1}{2} \inf _{\mathcal{C}(u)=x_{0}}\|u\|_{L_{2}}^{2}=\frac{1}{2}\left\|\mathcal{C}^{\dagger}\left(x_{0}\right)\right\|_{L_{2}}^{2}, \\
& L_{o}\left(x_{0}\right)=\frac{1}{2}\|y\|_{L_{2}}^{2}=\frac{1}{2}\|\mathcal{O}\|_{L_{2}}^{2},
\end{aligned}
$$

where $\arg \inf _{\mathcal{C}(u)=0} u=\mathcal{C}^{\dagger}\left(x_{0}\right)$, with $\mathcal{C}^{\dagger}: L_{2}^{m}\left(\mathbb{R}_{+}\right) \rightarrow \mathbb{R}^{n}$ representing the pseudo-inverse of $\mathcal{C}$. Under the assumptions 1.-3., $L_{c}\left(x_{0}\right)$ exist, $L_{c}>0, L_{c}(0)=0$ and satisfies the Hamilton Jacobi equation [2]:

$$
\frac{\partial L_{c}}{\partial x} f(x)+\frac{1}{2} \frac{\partial L_{c}}{\partial x} g(x) g^{T}(x) \frac{\partial^{T} L_{c}}{\partial x}=0
$$

such that $-\left(f(x)+g(x) g^{T}(x) \frac{\partial L_{c}(x)}{\partial x}\right)$ is asymptotically stable. If the system satisfies assumptions 1., 2. and 4. then $L_{o}$ exists, $L_{o}>0, L_{o}(0)=0$ and satisfies the nonlinear Lyapunov equation [2]:

$$
\frac{\partial L_{o}}{\partial x} f(x)+\frac{1}{2} h^{T}(x) h(x)=0 .
$$

We define the differential operators of $\mathcal{C}$ and $\mathcal{O}$ and their adjoints for (7). They correspond to the variational system and its Hamiltonian adjoint counterpart, both associated to
(7) (see [19], [3] for more details). We have:

$$
\begin{aligned}
& v=d \mathcal{C}(u)\left(u_{p}\right): \\
& \begin{cases}\dot{x}=-f(x)-g(x) u, & x(\infty)=0 \\
\dot{v}=-\frac{\partial(f(x)+g(x) u)}{\partial x} v-g(x) u_{p}, & v(\infty)=0 \\
v_{0}=v(0) & \end{cases} \\
& y_{p}=d \mathcal{O}\left(x_{0}\right)\left(v_{0}\right): \\
& \begin{cases}\dot{x}=f(x), & x(\infty)=0 \\
\dot{v}=\frac{\partial f(x)}{\partial x}, & v(\infty)=0 \\
y_{p}=\frac{\partial h(x)}{\partial x} v & \end{cases} \\
& y_{a}=(d \mathcal{C}(u))^{*}(p) \text { : } \\
& \begin{cases}\dot{x}=-f(x)-g(x) u, & x(\infty)=0 \\
\dot{p}=\frac{\partial^{T}(f(x)+g(x) u)}{\partial x} p, & p(0)=p_{0} \\
y_{a}=g^{T}(x) p & \end{cases} \\
& \begin{array}{c}
p_{0}=\left(d \mathcal{O}\left(x_{0}\right)\right)^{*}\left(u_{a}\right): \\
\begin{cases}\dot{x}=f(x), & x(\infty)=0 \\
\dot{p}=-\frac{\partial^{T} f(x)}{\partial x} p-\frac{\partial^{T} h(x)}{\partial x} u_{a}, & p(0)=p_{0} \\
p_{0}=p(0)\end{cases}
\end{array}
\end{aligned}
$$

We give a property relating $(d \mathcal{C}(u))^{*}(p)$ defined in $(13 \mathrm{c})$ to $\mathcal{C}^{\dagger}\left(x_{0}\right)$ :

Proposition 9: Let system (7) satisfy the assumptions 1.3 . and assume that $\mathcal{C}^{\dagger}$ exists and is continuously differentiable. Then

$$
y_{a}=\left(d \mathcal{C}\left(\mathcal{C}^{\dagger}\left(x_{0}\right)\right)\right)^{*}\left(\frac{\partial^{T} L_{c}}{\partial x}\right)=\mathcal{C}^{\dagger}\left(x_{0}\right) .
$$

Proof: First, according to [3], if $\mathcal{C}^{\dagger}$ exists and is continuously differentiable, then $\mathcal{C}^{\dagger}$ has the following state-space representation:

$$
\begin{aligned}
& \dot{x}=-f(x)-g(x) g^{T}(x) \frac{\partial^{T} L_{c}}{\partial x}, \quad x_{0}=x(0) \\
& y_{a}=g^{T}(x) \frac{\partial^{T} L_{c}}{\partial x} .
\end{aligned}
$$

Denote by $p=\frac{\partial^{T} L_{c}(x)}{\partial x}$. The dynamics of $p$ with respect to $\dot{x}=-f(x)-g(x) u$ is $\dot{p}=\frac{d}{d t}\left(\frac{\partial^{T} L_{c}(x)}{\partial x}\right)=$ $-\frac{\partial^{2} L_{c}(x)}{\partial x^{2}}(f(x)+g(x) u)$. For $u=g^{T}(x) \frac{\partial^{T} L_{c}(x)}{\partial x}$, the input that defines $L_{c}(x)$ we get:

$$
\dot{p}=-\frac{\partial^{2} L_{c}(x)}{\partial x^{2}}\left(f(x)+g(x) g^{T}(x) \frac{\partial^{T} L_{c}(x)}{\partial x}\right) .
$$

Since the system is under the assumptions 1.-3., then $L_{c}(x)$ exists and satisfies (11). Taking the derivative with respect to $x$ in (11) we get:

$$
\begin{aligned}
\frac{\partial^{2} L_{c}}{\partial x^{2}} f(x) & +\frac{\partial^{T} f}{\partial x} \frac{\partial^{T} L_{c}}{\partial x}+\frac{\partial^{2} L_{c}}{\partial x^{2}} g(x) g^{T}(x) \frac{\partial^{T} L_{c}}{\partial x} \\
& +\sum_{i=1}^{m} \frac{\partial L_{c}}{\partial x} g_{i}(x) \frac{\partial\left(g_{i}^{T}(x)\right)}{\partial x} \frac{\partial^{T} L_{c}}{\partial x}=0 .
\end{aligned}
$$

Rearranging the terms we obtain the following:

$$
\begin{aligned}
& \frac{\partial^{2} L_{c}(x)}{\partial x^{2}}\left(f(x)+g(x) g^{T}(x) \frac{\partial^{T} L_{c}(x)}{\partial x}\right) \\
& =-\left(\frac{\partial^{T} f(x)}{\partial x}-\sum_{i=1}^{m} \frac{\partial L_{c}(x)}{\partial x} g_{i}(x) \frac{\partial\left(g_{i}^{T}(x)\right)}{\partial x}\right) \frac{\partial^{T} L_{c}(x)}{\partial x}
\end{aligned}
$$


which, by using (15) is equivalent to

$$
\dot{p}=\frac{\partial^{T}(f(x)+g(x) u)}{\partial x} p, \text { for } p=\frac{\partial^{T} L_{c}(x)}{\partial x} .
$$

Then by the definition of $d \mathcal{C}^{*}$ in (13c) $y_{a}=g^{T}(x) \frac{\partial^{T} L_{c}(x)}{\partial x}=$ $\left.g^{T}(x) p\right|_{p=\frac{\partial^{T} L_{c}(x)}{\partial x}}$, which proves the statement of the proposition.

Remark 10: In the linear case, if $W>0$ is the controllability Gramian, then it satisfies equation (3) which is equivalent to $W A^{T} W^{-1}=-\left(A+B B^{T} W^{-1}\right)$. Then by the definition of the adjoint of the controllability operator we have $\mathcal{C}^{*}\left(p_{0}\right)=B^{T} e^{A^{T} t} p_{0}=B^{T} e^{-W^{-1}\left(A+B B^{T} W^{-1}\right) t} W p_{0}$. By the definition of the pseudo-inverse of the controllability operator, we have

$$
\mathcal{C}^{*}\left(p_{0}\right)=\mathcal{C}^{\dagger}\left(W p_{0}\right)
$$

\section{B. Symmetry}

1) A gradient approach: A natural nonlinear extension of the notion of symmetric systems is the gradient systems. In [10] a system is gradient if its associated variational system and its gradient extension counterpart have the same I/O behaviour. Based on this characterization in [20] a crossGramian is defined for the variational system as a solution of a nonlinear Sylvester equation.

It is conjectured that the Hankel singular value problem is equivalent to solving an eigenvalue problem of the crossGramian $\mathcal{X}$. At this moment this conjecture is still difficult to prove, since we are yet unable to provide a link between the gradient extension and the adjoints of the derivative of the controllability and observability operators, which are directly related to the above mentioned singular value problem.

2) A state-space approach: In this section, we extend the results obtained in Proposition 6 giving a new definition of the symmetry property, its metric and the cross-operator for a nonlinear dynamical system in terms of relations between the operators $\mathcal{C}^{\dagger}$ (the pseudoinverse of the controllability operator), its derivative $d \mathcal{C}^{\dagger}$ and $\mathcal{O}$. We give a necessary and sufficient condition for a nonlinear system to be symmetric with a cross-operator. First we give a definition of symmetry from an input-output point of view:

Definition 11: Let a system (7) be under the assumptions 1.-4. We call the system symmetric if it satisfies

$$
\operatorname{Im} \mathcal{O}=\operatorname{Im} \mathcal{C}^{\dagger}
$$

Proposition 12: A system (7) is symmetric in the sense of Definition 11 if and only if there exists an invertible function $\Phi(x)$ such that

$$
\mathcal{O}(x)=\mathcal{C}^{\dagger}(\Phi(x)) \text { and } \Phi(x)=\mathcal{C O}(x)
$$

or if there exists a smooth function $\Psi(\xi)$ such that:

$$
\mathcal{C}^{\dagger}(\xi)=\mathcal{O}(\Psi(\xi))
$$

with $\Psi(\xi)=\Phi^{-1}(\xi)$.
The proof follows from definition of an image of an operator and the symmetry property (16).

We call $\Phi$, defined in relation (17), the cross-operator of the symmetric system (7).

Remark 13: According to Section 1, a linear system $G(s)=C(s I-A)^{-1} B$ that is assumed minimal is symmetric if and only if $G(s)=G^{T}(s)$, which is equivalent to the existence of a metric $T$ s.t. the $(A, B, C)$ realization and its dual $\left(A^{T}, C^{T}, B^{T}\right)$ are state equivalent, as in Definition 1 if and only if relations (5) hold. It means, there exists $\Phi(x)=W T x=X x$ such that $\mathcal{O}(x)=\mathcal{C}^{\dagger}(W T x)$.

A cross-energy function is defined as

$$
L_{X}(x, \xi)=\frac{1}{2}\left\langle\mathcal{C}^{\dagger}(\xi), \mathcal{O}(x)\right\rangle .
$$

If the system is assumed symmetric with $\xi=\Phi(x)$ as in Proposition 12, using (17) we have:

$$
L_{X}(x, \Phi(x))=L_{X}(x)=\frac{1}{2}\langle\mathcal{O}(x), \mathcal{O}(x)\rangle=L_{o}(x)
$$

or

$$
L_{X}(\xi, \Psi(\xi))=L_{X}(\xi)=\frac{1}{2}\left\langle\mathcal{C}^{\dagger}(\xi), \mathcal{C}^{\dagger}(\xi)\right\rangle=L_{c}(\xi)
$$

and moreover

$$
L_{o}(x)=L_{c}(\Phi(x)) \text {. }
$$

Lemma 14: For a nonlinear symmetric system in the sense of Definition 11, the following property holds:

$$
(d \mathcal{O}(x))^{*} \mathcal{O}(x)=(d \Phi(x))^{*}\left(d \mathcal{C}^{\dagger}(\Phi(x))\right)^{*} \mathcal{C}^{\dagger}(\Phi(x))
$$

or, equivalently

$$
(d \Psi(\xi))^{*}(d \mathcal{O}(\Psi(\xi)))^{*} \mathcal{O}(\Psi(\xi))=\left(d \mathcal{C}^{\dagger}(\xi)\right)^{*} \mathcal{C}^{\dagger}(\xi) .
$$

The result is obtained by taking the derivative with respect to $x$ in the definition of $L_{o}$, in (10), and applying (19) and the chain rule of derivation.

Remark 15: If the linear system is symmetric then we have $\Phi(x)=W T x$, and equation (20) becomes $M x=$ $(T W) W^{-1}(W T x)$ which gives $M x=T^{T} W T x$ for all $x$. This means that the controllability gramian of the equivalent system $\left(T A T^{-1}, T B, C T^{-1}\right)$ is the observability Gramian of $(A, B, C)$. Since the symmetry property is assumed, $\left(T A T^{-1}, T B, C T^{-1}\right)=\left(A^{T}, C^{T}, B^{T}\right)$ with matrix $T$ symmetric and then $T^{-1} M x=W T x$ which is one of the definitions of the cross Gramian $X$ as in Theorem 5 .

Using Definition 11 for symmetry of the nonlinear system and the property in Proposition 9 we have a set of relations described by the diagram presented in Figure 1.

Since the system is assumed symmetric, according to Proposition 12, there exists $\xi=\Phi(x)$ as a coordinate transformation between $\mathcal{O}(x)$ and $\mathcal{C}^{\dagger}(\xi)$. Also, Proposition 9 , establishes a coordinate transformation between $\mathcal{C}^{\dagger}(\xi)$ and the operator $\left(d \mathcal{C}\left(\mathcal{C}^{\dagger}(\xi)\right)\right)^{*}(p)$, defined as $p=\frac{\partial^{T} L_{c}}{\partial \xi}(\xi)$. Then we can define

$$
\nu(x)=\frac{\partial^{T} L_{c}}{\partial \xi}(\Phi(x))=p
$$




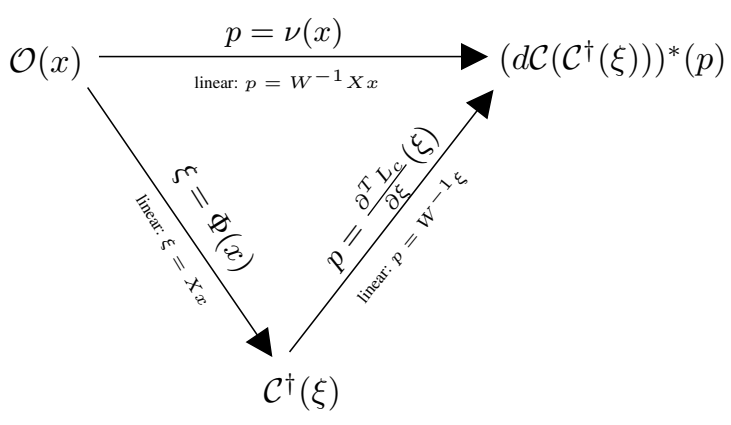

Fig. 1. Definition of symmetry.

as a coordinate transformation between $\mathcal{O}(x)$ and $\left(d \mathcal{C}\left(\mathcal{C}^{\dagger}(\xi)\right)\right)^{*}(p)$. This further yields:

$$
\Phi(x)=\left(\frac{\partial^{T} L_{c}}{\partial \xi}\right)^{-1}(p)=\left(\frac{\partial^{T} L_{c}}{\partial \xi}\right)^{-1}(\nu(x)) .
$$

Remark 16: In the linear case the symmetric matrix $T$ in Proposition 2 satisfies: $p=T x=W^{-1} W T x=W^{-1} X x$, where $X$ is the cross Gramian described by Theorem 5 .

Proposition 17: A nonlinear system is symmetric if and only if there exists $p=\nu(x)$ invertible and $\xi=\Phi(x)$ invertible such that

1)

2)

$$
\frac{\partial \nu(x)}{\partial x} f(x)=\frac{\partial^{T}\left(f(\xi)+g(\xi) g^{T}(\xi) p\right)}{\partial \xi} p
$$

and $h(x)=g^{T}(\xi) p$

$$
\begin{aligned}
& \frac{\partial \Phi(x)}{\partial x} f(x)=-f(\xi)-g(\xi) g^{T}(\xi) \frac{\partial^{T} L_{c}}{\partial \xi}(\xi) \\
& \text { and } h(x)=g^{T}(\xi) \frac{\partial L_{c}}{\partial \xi}(\xi)
\end{aligned}
$$

3) the functions $\Phi(x)$ and $\nu(x)$ satisfy relation (22) or (23).

Remark 18: The first statement of Proposition 17 is a nonlinear extension of the definition of symmetry: there exists a metric $p=T x$ such that $T A=A^{T} T, C=T B^{T}$. The second statement reads $X A=\left(A+B B^{T} W^{-1}\right) X$ and $X W^{-1} B=C^{T}$ which is equivalent to the Sylvester equation (2).

We give a nonlinear counterpart of the Sylvester equation (2):

Proposition 19: A nonlinear system is symmetric, if and only if $\Phi(x)$ satisfies:

$$
p^{T} \frac{\partial \Phi(x)}{\partial x} f(x)+\frac{1}{2} p^{T} g(\Phi(x)) h(x)=0,
$$

where $p$ satisfies (22) such that $h(x)=g^{T}(\Phi(x)) p$.

Remark 20: In the linear case we have: $p^{T} X A x+$ $\frac{1}{2} p^{T} B C x=0$.

\section{THE Singular VAlue ANALYSis OF THE HANKEL} OPERATOR: THE SYMMETRIC SYSTEMS CASE

\section{A. The Hankel singular value problem}

In this section we briefly describe the Hankel singular value problem for a nonlinear system as shown in the results from [3], [5], useful for obtaining a balanced realization. The Hankel operator is defined by the relation (9) and the starting point is the investigation of the gain structure of this operator. The gain structure problem means examining the largest singular value, where a singular value is defined as

$$
\sigma(u)=\frac{\|\mathcal{H}(u)\|_{L_{2}}}{\|u\|_{L_{2}}}
$$

The following problem must be solved, according to [5]:

$$
d \frac{\|\mathcal{H}(u)\|_{L_{2}}}{\|u\|_{L_{2}}} d u=0, \text { subject to } u \in \operatorname{Im} \mathcal{C}^{\dagger},
$$

that characterizes all the critical points $u$ as well as the optimal one that gives the largest eigenvalue. According to [3], [5] this problem has the alternative formulation: there exists $\lambda \in \mathbb{R}$ s.t.

$$
(d \mathcal{H}(u))^{*} \mathcal{H}(u)=\lambda u .
$$

The problem of finding $u \in \operatorname{Im} \mathcal{C}^{\dagger}$ such that equation (27) is satisfied, is called the Hankel singular value problem. An alternative and simpler characterization of the equation (27) is described in the following.

Theorem 21: [5] Assume that the controllability operator, its pseudo-inverse and the observability operator exist and are continuously differentiable. Assume, moreover that there exists $\lambda \in \mathbb{R}$ and $x \in \mathbb{R}^{n}$ satisfying

$$
d L_{o}(x)=\lambda d L_{c}(x) .
$$

Then if

$$
u \in \operatorname{Im} \mathcal{C}^{\dagger}
$$

it satisfies the Hankel singular value equation (27).

From the definitions of $L_{c}(x)$ and $L_{o}(x)$ in (10) equation (28) can be rewritten equivalently as:

$$
(d \mathcal{O}(x))^{*} \mathcal{O}(x)=\lambda\left(d \mathcal{C}^{\dagger}(x)\right)^{*} \mathcal{C}^{\dagger}(x) .
$$

Remark 22: In the linear case, equation (28) is equivalent to $x^{T} M=\lambda x^{T} W^{-1}$, which is similar to: $W M x=\lambda x$, i.e. the squared Hankel singular values are the eigenvalues of $W M$.

\section{B. The symmetric case}

Under the assumption of symmetry upon the system, the Hankel singular value equation (27) or (30), can be written in terms of the cross-operator and the derivative of one of the energy functions.

Theorem 23: Assume a nonlinear system (7) is symmetric in the sense of Definition 11. The differential eigenstructure problem for (7) becomes

$$
\frac{1}{\lambda} p=\left(\frac{\partial \Phi}{\partial x}\left(\nu^{-1}(p)\right)\right)^{-1} \cdot \frac{\partial L_{c}}{\partial x}\left(\nu^{-1}(p)\right)
$$

or, equivalently

$$
\frac{1}{\lambda} p=\frac{\partial^{T} L_{o}}{\partial \xi}(\xi),
$$


where $\xi$ is described as $\xi=\left(\Phi \circ \nu^{-1}\right)(p)$. Moreover $\lambda$ is identical to the one in (27).

Proof: Starting by the definition of the largest Hankel singular value subject to the constraint $u \in \operatorname{Im} \mathcal{C}^{\dagger}$ as the reasoning in [5]. Due to the symmetry property, from relation (18)

$$
\|\mathcal{H}(u)\|_{L_{2}}=\langle\mathcal{O}(x), \mathcal{O}(x)\rangle^{\frac{1}{2}}=\left\langle\mathcal{C}^{\dagger}(\Phi(x)), \mathcal{C}^{\dagger}(\Phi(x))\right\rangle^{\frac{1}{2}} .
$$

Then we evaluate $d \frac{\left\langle\mathcal{C}^{\dagger}(\Phi(x)), \mathcal{C}^{\dagger}(\Phi(x))\right\rangle^{\frac{1}{2}}}{\left\langle\mathcal{C}^{\dagger}(x), \mathcal{C}^{\dagger}(x)\right\rangle^{\frac{1}{2}}} d x=0$. Computing the derivative in the left term we have

$$
\begin{aligned}
& \frac{\left\langle\mathcal{C}^{\dagger}(x), \mathcal{C}^{\dagger}(x)\right\rangle^{\frac{1}{2}}}{\left\langle\mathcal{C}^{\dagger}(\Phi(x)), \mathcal{C}^{\dagger}(\Phi(x))\right\rangle^{\frac{1}{2}}} \cdot\left\langle d \mathcal{C}^{\dagger}(\Phi(x)) d \Phi(x) d x, \mathcal{C}^{\dagger}(\Phi(x))\right\rangle \\
& -\frac{\left\langle\mathcal{C}^{\dagger}(\Phi(x)), \mathcal{C}^{\dagger}(\Phi(x))\right\rangle^{\frac{1}{2}}}{\left\langle\mathcal{C}^{\dagger}(x), \mathcal{C}^{\dagger}(x)\right\rangle^{\frac{3}{2}}} \cdot\left\langle d \mathcal{C}^{\dagger}(x) d x, \mathcal{C}^{\dagger}(x)\right\rangle=0
\end{aligned}
$$

Then applying the adjoint properties of $d \mathcal{C}^{\dagger}, d \mathcal{O}$, and denoting by $\lambda=\frac{\left\langle\mathcal{C}^{\dagger}(\Phi(x)), \mathcal{C}^{\dagger}(\Phi(x))\right\rangle}{\left\langle\mathcal{C}^{\dagger}(x), \mathcal{C}^{\dagger}(x)\right\rangle}$ and we have:

$$
\left\langle d x,(d \Phi(x))^{*}\left(d \mathcal{C}^{\dagger}(\Phi(x))\right)^{*} \mathcal{C}^{\dagger}(\Phi(x))-\lambda\left(d \mathcal{C}^{\dagger}(x)\right)^{*} \mathcal{C}^{\dagger}(x)\right\rangle=0,
$$

which yields:

$$
(d \Phi(x))^{*}\left(d \mathcal{C}^{\dagger}(\Phi(x))\right)^{*} \mathcal{C}^{\dagger}(\Phi(x))=\lambda\left(d \mathcal{C}^{\dagger}(x)\right)^{*} \mathcal{C}^{\dagger}(x)
$$

Applying (20), equation (33) becomes (30) which is as in Theorem 21 the Hankel singular value equation. (33) is equivalent to:

$$
\frac{\partial^{T} \Phi}{\partial x}(x) \frac{\partial^{T} L_{c}}{\partial x}(\Phi(x))=\lambda \frac{\partial^{T} L_{c}}{\partial x}(x),
$$

or, using (22) we obtain the first relation in the proposition. Equation (34) has an equivalent in terms of $L_{o}$. First, (22) is equivalent to:

$$
p=\frac{\partial^{T} \Psi(\xi)}{\partial \xi}(\xi) \frac{\partial^{T} L_{o}}{\partial \xi}(\Psi(\xi)) .
$$

The differential eigenstructure problem becomes:

$$
(d \Psi(\xi))^{*}(d \mathcal{O}(\Psi(\xi)))^{*} \mathcal{O}(\Phi(\xi))=\lambda(d \mathcal{O}(\xi))^{*} \mathcal{O}(\xi),
$$

which is equivalent to:

$$
\frac{\partial^{T} \Psi}{\partial \xi}(\xi) \frac{\partial^{T} L_{o}}{\partial \xi}(\Psi(\xi))=\lambda \frac{\partial^{T} L_{o}}{\partial \xi}(\xi),
$$

and yields (32).

Remark 24: In the linear case, we have: $\frac{1}{\lambda} p=$ $(T W)^{-1} W^{-1} T^{-1} \Leftrightarrow p^{T} \frac{1}{\lambda}=p^{T} X^{-2}$ which is the same as $p^{T} X^{2}=p^{T} \lambda$, consistent with Theorem 5 .

\section{CONCLUSIONS}

In this paper, a new symmetry notion and a cross-operator notion have been defined in terms of the controllability and observability operators. This is a nonlinear extension to the symmetric linear case and the cross-Gramian, respectively. We gave a necessary and sufficient condition for a nonlinear system to be symmetric and the cross-operator to be the solution of a nonlinear Sylvester-like equation. Then, we studied the Hankel singular value problem for symmetric systems and we gave a simpler form of the state equation that characterizes this problem, in terms of the cross-operator and the derivative of the controllability/observability function. For future work we want to provide a link between the gradient systems and the symmetric systems defined in this paper. Moreover, an extension the passive case is an open question.

\section{ACKNOWLEDGMENT}

We thank NWO (Dutch Science Foundation) for supporting this research.

\section{REFERENCES}

[1] B. C. Moore, "Principal component analysis in linear systems: controllability, observability and model reduction," IEEE Trans. Autom. Contr., vol. 26, pp. 17-32, 1981.

[2] J. M. A. Scherpen, "Balancing for nonlinear systems," Systems \& Control Letters, vol. 21, pp. 143-153, 1993.

[3] K. Fujimoto and J. M. A. Scherpen, "Nonlinear input-normal realizations based on differential eigenstructure of Hankel operators," IEEE Trans. Autom. Contr., vol. 50, pp. 2-18, 2005.

[4] E. Verriest and W. S. Gray, "Nonlinear balanced realizations," in Proc. 43rd IEEE Conf. on Decision and Control, 2004, pp. 1164-1169.

[5] K. Fujimoto and J. M. A. Scherpen, "Balanced realization and model order reduction for nonlinear systems based on singular value analysis," 2009, submitted.

[6] K. Zhou, J. C. Doyle, and K. Glover, Robust optimal control. Prentice Hall, 1996.

[7] N. Wong, "Efficient positive-real balanced truncation of symmetric systems via cross Riccati equations," IEEE TCAD, vol. 27, no. 3, pp. 470-480.

[8] W. Q. Liu, V. Sreeram, and K. L. Teo, "Model reduction for statespace symmetric systems," Systems \& Control Letters, vol. 34, pp. 99-113, 1998.

[9] B. Vanluyten and J. C. Willems, "Model reduction of systems with symmetries," in Proc. 44th IEEE CDC-ECC05, 2005, pp. 826-831.

[10] J. Cortes, A. J. van der Schaft, and P. E. Crouch, "Characterization of gradient control systems," SIAM J. Control \& Optimization, vol. 44, pp. 1192-1214, 2006.

[11] T. C. Ionescu and J. M. A. Scherpen, "Nonlinear cross Gramians," in Proc of the 23rd IFIP TC7, 2008, pp. 323-338.

[12] K. V. Fernando and H. Nicholson, "On the structure of balanced and other principal representations of SISO systems," IEEE Trans. Autom. Contr., vol. 28, no. 2, pp. 228-231, 1983.

[13] - "On a fundamental property of the cross-Gramian matrix," IEEE Trans. Circuits \& Systems, vol. 31, no. 5, pp. 504-505, 1984.

[14] _ "On the cross-Gramian for symmetric MIMO systems," IEEE Trans. Circuits \& Systems, vol. 32, no. 5, pp. 487-489, 1985.

[15] R. W. Aldaheri, "Model reduction via Schur-form decomposition," vol. 53, no. 3, pp. 709-716, 1991.

[16] D. C. Sorensen and A. C. Antoulas, "The Sylvester equation and approximate balanced truncation," Linear Algebra \& its Applications, vol. 351-352, pp. 671-700, 2002.

[17] J. C. Willems, "Dissipative dynamical systems - Part I: General theory, - Part II: Linear systems with quadratic supply rates," Archive for Rational Mechanics and Analysis, vol. 45, pp. 321-393, 1972.

[18] J. M. A. Scherpen, "Balancing for nonlinear systems," Ph.D. dissertation, Univ. of Twente, 1994.

[19] K. Fujimoto and J. M. A. Scherpen, "Singular value analysis of Hankel operators for general nonlinear systems," in Proceedings ECCO3, 2003.

[20] T. C. Ionescu and J. M. A. Scherpen, "Nonlinear cross Gramians and gradient systems," in Proc. 46th IEEE Conf. on Decision and Control, 2007, pp. $3745-3750$. 